\title{
Case Series: Various Radiologic Findings of Peritoneal Tuberculosis Mimicking Malignancy and Pathological Confirmation
}

\author{
Teuku Muhammad Yus, MD, , Muhammad Hidayat Surya Atmaja, MD \\ a Department of Radiology, Dr. Soetomo General Academic Hospital, Faculty of Medicine, Universitas Airlangga, Surabaya, Indonesia \\ ${ }^{b}$ Faculty of Medicine, Universitas Syiah Kuala, Banda Aceh, Indonesia \\ Corresponding email: teukuyus@unsyiah.ac.id
}

\begin{abstract}
Background: PTB is the common case found in many endemic countries. The misdiagnosis of PTB is often happening because it mimics malignancy and is difficult to distinguish from another infection.

Cases Presentation: Here presented three PTB cases, a male 23 years old, and two female patients 16 and 32 years old, respectively. The patients were initially suspected of intra-abdominal malignancy and finally confirmed as PTB after CT Scan and pathological examination. After several months of OAT treatment, the patient improved then the complaints are decreased. Conclusion: PTB is a disease that closely resembles a malignancy such as peritoneal carcinomatosis, and is difficult to distinguish from another infection. An adequate understanding of PTB characteristics on CT scans will be very helpful in establishing the diagnosis of these cases
\end{abstract}

Keywords: Peritoneal tuberculosis, Ascites, Peritoneal carcinomatous, Imaging, Mimicking

\section{Introduction}

Peritoneal TB (PTB) is a manifestation of TB infection that spreads to the abdominal cavity $(2,3)$. Intraabdominal infection of TB could be spreading of the originating infection foci in lungs or another location (3). Sometimes PTB is also called intestinal TB (4). The clinical appearances of PTB are sometimes non-specific and can resemble malignancy. To distinguish PTB from mimicking malignancy or other infections is the challenge. Radiologists play a significant role in this situation (5). Radiologists need to be aware of suspects of malignancy on young adult patients. Because base on malignancy incidents, there are quite rare on a young adult patient, although it could be happening (6). CT Scan is a very important modality to establish the diagnosis of PTB (7). However, PTB can deceive clinicians and cause misdiagnosis (8). Several reports state that the abdominal enlargement of reproductive age women is sometimes suspected of ovarian cancer, this is related to the clinical symptoms that appear (5). The radiologist should to be realize that PTB as one of the differential diagnoses of any young adult patients with intra-abdominal mass, even the $\mathrm{CT}$ scan findings are mimicking malignancy (8).

\section{Case 1}

A male 23-year-old referred to emergency department with severe colic abdominal pain at the upper left abdominal region since in the last month. The patient also complaint about abdominal enlargement, sometimes accompanied by sub-febrile fever in the last 3 months. There is no history of tuberculosis. He is a smoker and drinking alcohol. Defecation is not so smooth and urination is normal. The laboratory examination results are mild anaemic, increase of leukocytes count are $12.000 / \mathrm{mcl}$, increase the lactate dehydrogenase (LDH) levels of 282 $\mathrm{U} / \mathrm{L}, \mathrm{C}$-reactive protein (CRP) $6.3 \mathrm{mg} / \mathrm{dl}$, and normal alpha-fetoprotein (AFP) level. Chest $\mathrm{x}$-ray is normal, abdominal x-ray shows bowel dilatation suggesting obstructive ileus. The patient then underwent the contras abdominal CT scan with the findings are multiple lymph nodes enlargement in the abdominal region, conglomerated and forming the mass at the epigastrium region, diffuse bowel wall thickening, and the lesion are enhancing. No, ascitic fluid founded. The CT scan suggesting as PTB (fig.1). Then the patient underwent core biopsy procedure for pathologic examination. The pathologic result are large necrotic areas with infiltration of lymphocyte inflammatory cells, and groups of epithelioid cells that vaguely formed granulomas, no signs of malignancy were seen. With Ziehel Neelsen's staining, the Mycobacterium tuberculosis was obtained. Pathologic examination was concluded as chronic granulomatous inflammation according to tuberculosis (fig.1). Then, the patient given category 2 of anti-tuberculosis (OAT) planned for 3 months. It is consisting of ethambutol, isoniazid, rifampicin, and pyrazinamide. The patient's getting better after 2 months follow up during the therapy, the OAT therapy continued. 


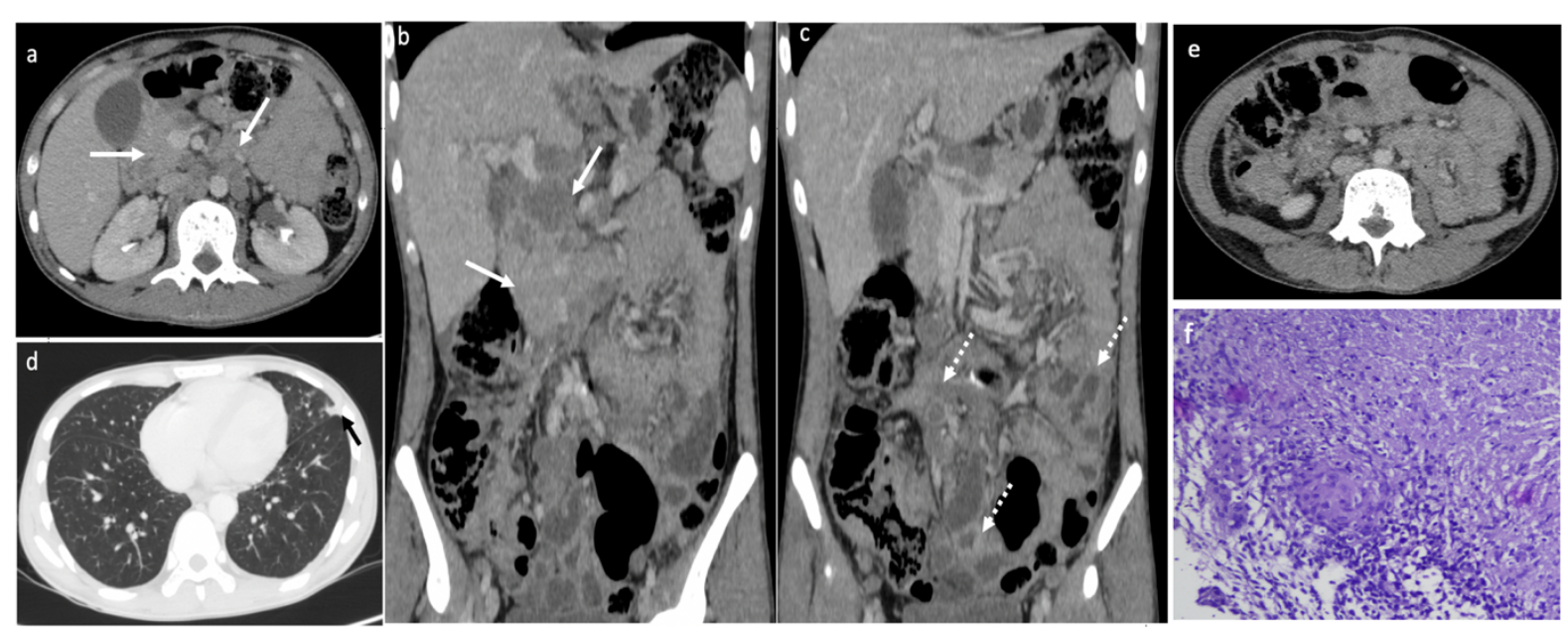

Figure 1. Abdominal contrast CT scan, axial and coronal view of the portal vein phase (a, b, c) bowel wall thickening (white arrows) and (b) paraaortic nodules (white arrows) conglomerate to form a mass accompanied by (c) thickening of the bowel wall (dotted arrow), (d) fibrosis at the anterior basal of the left lung (black arrow), possibly post tuberculosis infection. (f) H\&E (200x), there is large areas of necrosis and clusters of epithelioid cells vaguely form granulomas accompanied by foci of caseous necrosis and Datia-Langerhans cells, Ziehl-Neelson staining showed Mycobacterium tuberculosis. No signs of malignancy.

\section{Case 2}

A girl, 16 years old present to paediatric outpatient with complaints of abdominal enlargement accompanied by nausea and vomiting for the last 2 months. She felt a lump on the left side of her waist and a significant loss of body weight. She had a history of pulmonary TB, received 6 months of OAT treatment and had been declared cured by a clinician since 1 last year. Currently, occasionally with intermittent mild fever. The menstrual cycle normal without any abnormal vaginal bleeding. After blood laboratory examination, there was slight increase of leukocytes count are $10.500 / \mathrm{mcl}$, mild anaemic $11 \mathrm{mg} / \mathrm{dl}$, and a slight decrease of albumin level. Liver function is normal. The paediatrician request for abdominal contrast CT Scan. The abdominal contrast CT scan findings are high density of the ascitic fluid (18-22 HU), there are also multiple cystic with thick fluid contain, those lesions were enhancing on contrast administration. There were also found multiple mesenteric nodules with the biggest size are $1.3 \times 1.6 \mathrm{~cm}$, and multiple lymph nodes at the para-aortic region. Those findings were concluded as suggested of PTB (fig.2), and need further confirmation by pathological examination. The pathological examination (fig.2) by laparoscopic biopsy procedure for pathologic specimen obtained. The findings are pieces of fibrous connective tissue without stratified epithelial cells with large necrotic areas surrounded by epithelioid histiocyte cells forming granulomas, in accordance with the picture of TB. No signs of malignancy. The patient received therapy of OAT category 2 (ethambutol, isoniazid, rifampicin, and pyrazinamide) planned for 6 months. On follow-up after 3 months of the treatment, the abdominal enlargement began to shrink and complaints improved.
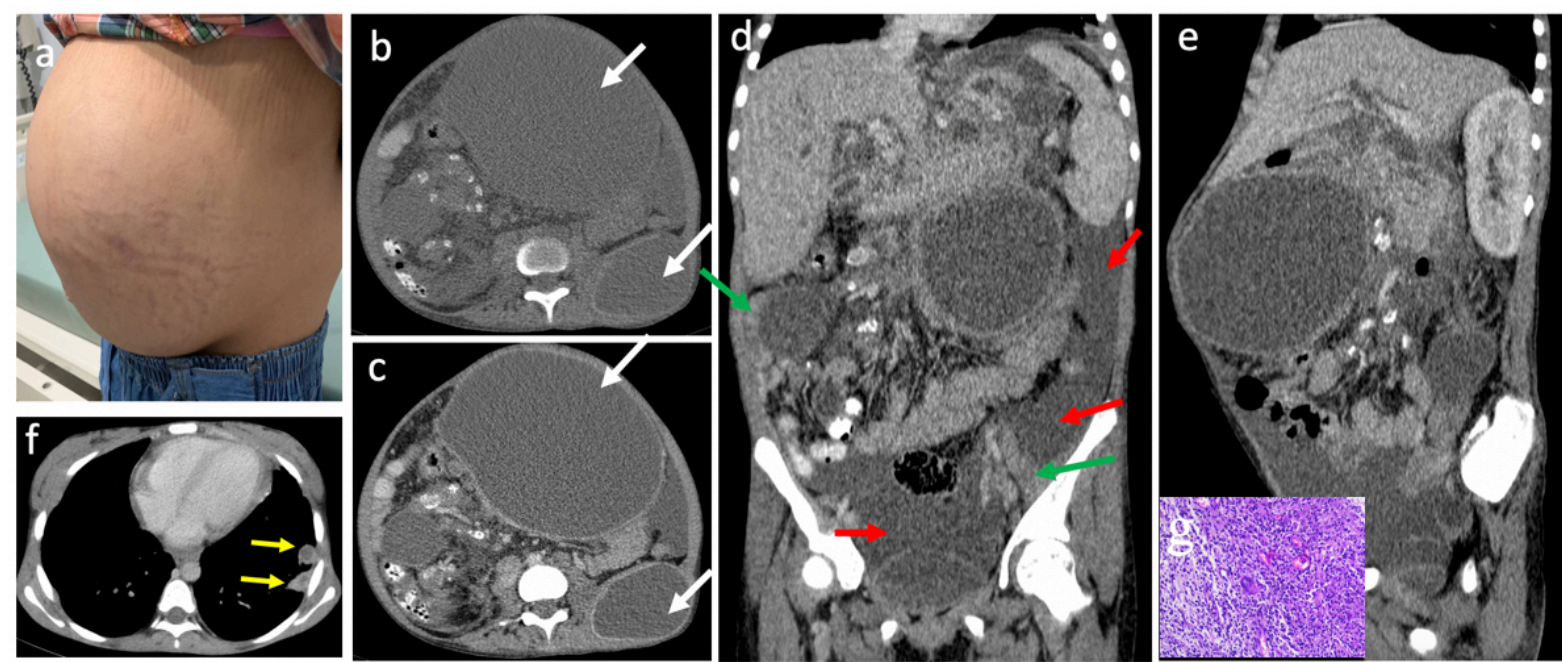

Figure 2. A girl 16 years old, with abdominal enlargement according to clinical appearance (a), non-contrast CT scan, axial view (b) the free fluid of ascites and multiple loculated ascites, then after being given the contrast at venous phase (c) the wall/septa with contrast enhancement (white arrows), (d) coronal view of the venous phase; Thickening of the bowel wall is seen in the right superolateral and left inferrolateral 
abdominal cavity (green arrows) and ascites (red arrows), (e) the coronal view shows that the left kidney is pushed to the superior-posterior, (f) subpleural abscess with rim contrast enhancement in the venous phase. (g) H\&E (20x), visible pieces of fibrous connective tissue without stratified epithelial cells, a large necrotic area surrounded by epithelioid-shaped histiocytes cells which then form granulomas, these findings are in accordance with peritoneal tuberculosis (PTB)

\section{Case 3}

A young adult woman, 32 years old, present to gynaecology outpatient with complaint of abdominal enlargement and pain for the last 5 months, intermittent fever, no history of TB before. Patient married and have 2 kids, irregular menstrual cycles. The clinician's suspected of the malignant ovarian tumour with ascites. Then she underwent the laboratory examination with the result: increase of SGOT and SGPT levels, normal leukocyte count, CEA level $0.34 \mathrm{ng} / \mathrm{ml}, \mathrm{CA}-125$ are $53.7 \mathrm{U} / \mathrm{ml}$, normal AFP, and normal LDH levels. The albumin level is normal. Then the gynaecologist request for abdominal contrast CT Scan. The results of a CT scan of the abdomen were obtained, free ascites with thick density in the abdominal cavity accompanied by thickening of part of the bowel wall, multiple lymph nodes were also found in the mesentery. Both adnexa looked fine. Hematometra is seen in the uterine cavity. The results of a CT scan of the abdomen concluded as PTB (fig.3). Subsequently, a biopsy has been performed by laparoscopic procedure for pathological examination, the results obtained are fibrous connective tissue, fatty tissue, epithelioid histiocyte cells that form granulomas with Datia-Langerhans cells. There were no signs of malignancy in this patient. The pathological examination concluded as granulomatous inflammation according to the picture of tuberculosis (fig.3). The patient was given OAT therapy (rifampicin, ethambutol, and azithromycin). During the follow-up evaluation after 3 months later, the patient has been improving, the abdominal becomes smaller, and the pain was reduced. After that, OAT treatment is continued for up to 6 months.
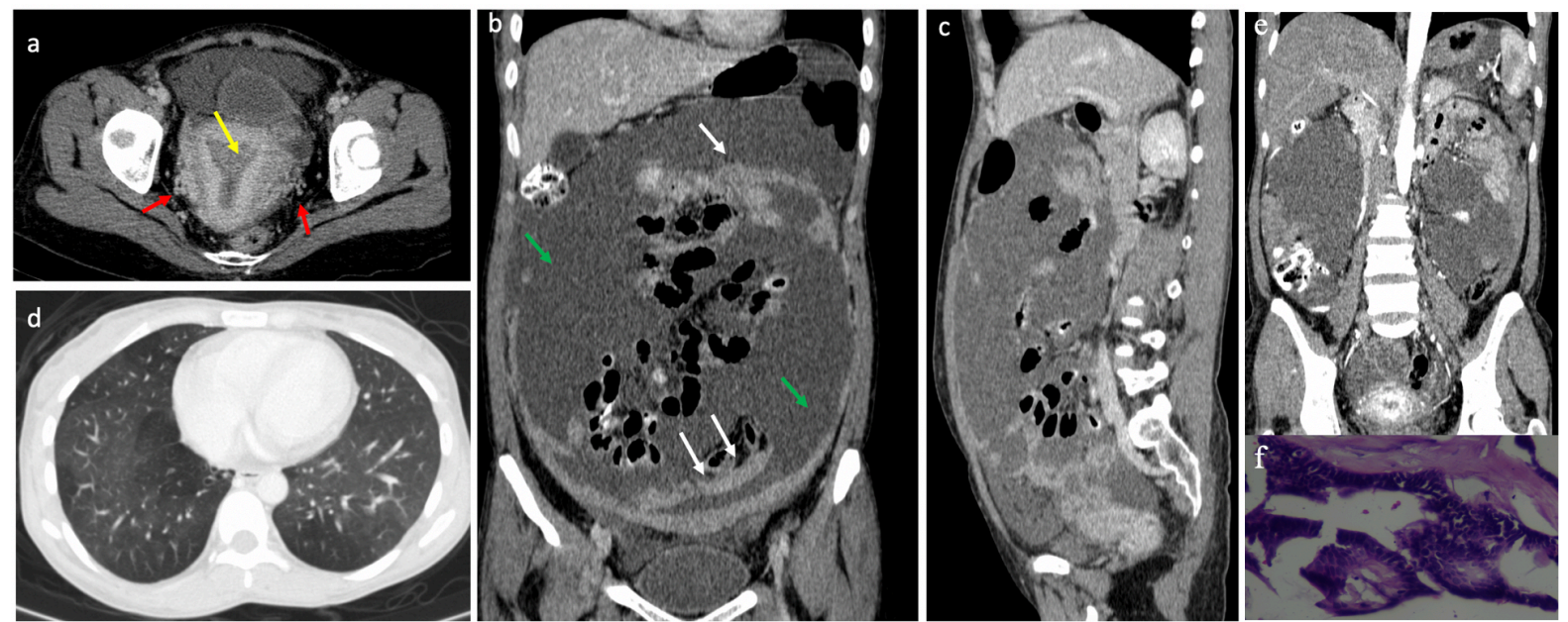

Figure 3. Female, 32-year-old, an abdominal CT scan with iodine contrast administration; (a) Axial view of the venous phase; there is a dilatation of uterine cavity filled by blood (hematometra; yellow arrow), no any adnexal mass, as well as multiple sub-centimetre lymphadenopathies at the right iliac region (red arrow), (b) coronal view of venous phase (c sagittal view of venous phase; severe ascites is seen throughout the abdominal cavity and pelvis (green arrows), bowel wall thickening also seen in the inferior and superior abdominal regions (white arrows), (d) on Axial view of CT scan at the lung window, no nodules or consolidation seen in the right and left lung bases, (e) coronal view of arterial phase, (f) H\&E (200X), a large area of necrotic found with groups of epithelioid cells that vaguely formed a granuloma, accompanied by caseous necrotic

\section{Discussion}

The recent advances in medical science and technology are supposed to resolve various health problems (9). Various health problems that used to be serious may be handled or avoided recently. However, tuberculosis (TB) which was discovered by Robert Koch in 1882, today remains to be a serious problem worldwide, especially in tropical countries as endemic areas. So many treatment programs have been implemented since previous decades (3). Despite this, TB still a serious problem today. This may relate to the increase of populations, and immunocompromised problems, particularly in tropical countries $(9,10)$. Based on WHO report 2019, TB remains one of the most common causes of death worldwide. Estimated 10 million TB cases causing 1.2 million deaths of TB in 2019, the addition deaths about 208,000 cases of HIV with TB. The global distribution of TB includes Southeast Asia (44\%), Africa (25\%), and the Western Pacific (18\%), while smaller percentages in the Eastern Mediterranean (8.2\%), Americas (2.9\%), and Europe (2.5\%) (9). Currently, two-thirds of the world's TB incidence 
carry out from 8 countries, such as India (26\%), Indonesia (8.5\%), China (8.4\%), Philippines (6.0\%), Pakistan (5.7\%), Nigeria (4.4\%), Bangladesh (3.6\%), and South Africa (3.6\%) (9).

The most common finding of TB is in the respiratory system. However, other organ systems may be infected by TB, such as the brain, bones, intestinal, urinary tract, and reproductive organs (6). The incidence of abdominal TB is reported $11-12 \%$ of all of the extrapulmonary TB cases. PTB is the most common clinical manifestation of abdominal tuberculosis as generalized (11).

Specifically, PTB is divided into three main types, namely: wet, fibrotic, and dry types. CT scan could distinguish the types, although third types can be overlapping and difficult to distinguish (12). The wet type of PTB is the most common type of peritonitis TB, usually with free or localized ascites. The ascitic fluid of PTB relatively has a higher attenuation than other infections. Because the ascitic fluid of PTB contains high protein and high cellular components $(3,12)$. Fibrotic type PTB is characterized by the formation of omental or mesenteric nodal, both components appear to be omental thickening like "cake" mixed with webbing of intestinal loops that called the "omental cake" sign. On CT scan it will appear as a low attenuation mottled mass with a nodular soft tissue thickening $(5,12)$. The thickening tissue and "omental cake" sign can also be evaluated by ultrasonography. Furthermore, dry-type PTB is a rare case characterized by mesenteric thickening, fibrous adhesions, and caseous nodules $(12,13)$. Radiologic examination especially CT Scan is highly recommended to differentiate both abnormalities. But radiologic examination is not specific to differentiate PTB from others infections $(7,12)$.

The accuracy of radiological examination is needed to establish a diagnosis of the several PTB cases mentioned above (6). The $\mathrm{x}$-ray modality was reported as a minimum contribution in establishing the diagnosis of PTB. The findings of plain abdominal x-ray sometimes only show local ileus, ascites, and sometimes a mass that is not specific $(3,6)$. Meanwhile, Ultrasonography (USG) can identify a bowel wall thickening, ascites, and mesenteric nodules, but it is not always as well as easy, and sonography is very operator-dependent (6).

The advanced imaging of CT Scan is believed to be highly recommended and very informative. CT scan will be able to differentiate masses or conglomeration of an intra-abdominal lymph node, assess the condition of the bowel wall, and detect minimal ascites, including assessing the viscosity of the ascitic fluid itself by hustleunit (HU) level $(7,14)$. The ascites fluid viscosity can help differentiate the ascites that carry out by malignant process or due to infections, including mycobacterium tuberculosis infection $(5,7)$. However, a CT scan is insufficient to evaluate the abnormalities of intestinal mucosal such as ulceration or stricture (5). There are several abdominal CT Scan findings that support the PTB appearances, are:

1. Lymphadenopathy; intra-abdominal lymph node enlargement is the most common manifestation of PTB $(4,10)$. However, the same pathological also occur in several other diseases, such as lymphoma and other malignancy or infections. PTB is certainly associated with enlargement of lymph nodes, despite it being rarely associated with peritoneal and other solid organs abnormalities $(10,15)$. The most common lymph nodes that are affected include; mesenteric lymph nodes, omental lymph nodes, lymph nodes around the porta hepatica, celiac trunk, and also peripancreatic lymph nodes (15). The radiological image of lymph nodes associated with PTB can be various, an increase of the nodes number, lymph node enlargement, and conglomeration of lymph node and forming a mass-like appearance. The pathologic lymph nodes of PTB usually show a central caseous necrotic area and peripheral enhancement. The presence of calcification is also often associated with PTB $(13,15)$.

2. Ascites; ascites is also a common finding associated with PTB (2). Although the volume of ascitic fluid could be various, minimal or massive. Sometimes, it just only loculated ascites (8). Ascites on PTB are a thick exudate fluid with a density (20-45 HU) on a CT scan (4). It is related to the high protein content and high cellular components and is useful to differentiate ascites PTB or malignancies. The ascitic fluid of malignancy commonly transudates with low attenuation, or may also differ on other ascites $(4,5)$.

3. Peritoneal thickening; peritoneal thickening is a common finding in PTB (5). The peritoneal thickening associated with multiple small nodules will be better visualized if ascites is present (4). On PTB, the peritoneal 
appearance is usually with a smooth contour, minimal thickening, and enhancement after contrast administration. While the irregular peritoneal thickening with nodular implants also can be the suggestion of peritoneal carcinoma (8).

4. Omental thickening; anatomically, the omental tissue is at the superficial and anterior sides of the bowel (16). The dilatation and distention of the lumen bowel will be clear on CT scan images. But in the case of lumen collapse, it could be different. The features of omental involvement in PTB will be identified by multiple omental nodular, and it has been reported in many cases of PTB $(8,15)$.

5. Small Intestine Mesenteric; small bowel mesenteric is a part of the peritoneum. Small bowel mesenteric involvement also has been reported in the majority of PTB cases (13). The most common mesenteric abnormality are multiple nodular lesions, mesenteric thickening, and loss of normal mesenteric configuration. Mesenteric nodular lesions may be micro $(<5 \mathrm{~mm})$ or macro $(>5 \mathrm{~mm})$, solid, cystic, or even abscess. By CT scan, the mesenteric involvement of PTB will be a thickening and high vascularity $(4,5)$. It can also be seen assembling the small bowel mesentery by pulling the intestinal loop forming a mass in the abdominal cavity $(8)$

The spreading possibility of tuberculosis infection to the intra-abdominal can be explained in several routes (17). The first route is the mycobacterium tuberculosis directly entering into the digestive tract, it is through the food or drinks that were contaminated by mycobacterium tuberculosis spores. Some of the spores can survive from gastric acid and pass a gastric, thereby infecting the intestinal mucosa. Furthermore, the germs or spores that enter will stick to the gastrointestinal mucosal layer and an infection process occurs, then forming an epithelioid tubercle in the intestinal submucosal lymphoid tissue $(13,17)$. Furthermore, after $2-4$ weeks, the focus of infection will develop to be caseous necrotic of the tubercles (17). This process can also cause ulceration of the gastrointestinal mucosa. The next process is the spread of infection to the lymph nodes around the bowel and peritoneum $(8,17)$. The spreads of this infectious process rarely directly into the portal or hepatic artery circulation, so the findings of solid organs infections such as liver, pancreas, and spleen are uncommon (17).

The second route, namely hematogenous spread. It is from the focus of infection (tuberculosis) in the lungs or other organs through the hematogenous route to the intra-abdominal organs. Some experts believe this path is considered to be the most common portion. This pathway allows the focus of intra-abdominal tuberculosis infection could to occur in solid abdominal organs $(2,17)$.

The third route, is the direct spreading of adjacent infection foci to the peritoneum. This infection foci may to spread reaching gynaecological organs such as fallopian tubes and ovaries. It has even been reported the spreading to psoas muscle or even to the vertebrae, those are causing tuberculous spondylitis. Otherwise, the infection foci in the vertebrae spread to psoas muscles and also infecting the gynaecological organs $(13,17)$.

The fourth way, namely the lymphogenic pathway. The spreading of tuberculosis infection focuses on the lymphatics and causes the enlargement of the infected lymph nodes, surely including intra-abdominal lymph nodes $(10,13)$.

\section{Differential Diagnosis}

1. Peritoneal carcinomatosis, it is also known as peritoneal metastasis, is a common site for intra-abdominal metastases, especially the metastatic of the abdominal and pelvic organs malignancies. Peritoneal carcinomatosis generally implies a poor prognosis. The presence of a primary malignant mass at another part will be the point that differentiates peritoneal carcinomatous from PTB. However, often the predominantly cystic adnexal mass is quite difficult to define as a primary mass or ascites (7).

2. Primary peritoneal mesothelioma, it is a rare primary malignancy that originates from the peritoneal lining. Abdominal primary mesothelioma is different from pleural primary mesothelioma. There are two sub-types of abdominal mesothelioma are multi-cystic mesothelioma and tunica vaginalis testis mesothelioma. In both 
of those cases, the patient usually presents with testicular enlargement, recurrent hydrocele, scrotal mass, and inguinal hernia (7).

\section{Conclusion}

Peritoneal tuberculosis (PTB) is a disease that closely mimics peritoneal carcinomatosis as a malignancy in the abdominal cavity. It is also difficult to distinguish from another infection. CT scans have high accuracy and reliability as the imaging modality of choice in distinguishing PTB. The CT Scan will be able to differentiate PTB from peritoneal carcinomatosis, primary abdominal mesothelioma, and help to differentiate other infections. Understanding the characteristics of PTB on CT scans will be very helpful in establishing an accurate diagnosis and avoiding misdiagnosis.

\section{Acknowledgement}

\section{Financial Support}

This research received no specific grant from any funding agency, commercial or not-for-profit sectors.

\section{Conflict of interest}

None.

\section{Ethical Standard}

None.

\section{Department and Institution where work was done}

The work was done in the Department of Radiology Dr. Soetomo General Hospital, Surabaya, Indonesia.

Notes on patient consent case report

Written informed consent was obtained from parent(s). 


\section{References}

1. Fox AJ, Millar J, Raymond J, Pryor JC, Roy D, Tomlinson GA, et al. Dangerous advances in measurements from digital subtraction angiography: When is a millimeter not a millimeter? Am J Neuroradiol. 2009;30(3):459-61.

2. Debi U, Ravisankar V, Prasad KK, Sinha SK, Sharma AK. Abdominal tuberculosis of the gastrointestinal tract: Revisited. World J Gastroenterol. 2014;20(40):14831-40.

3. Rathi P, Gambhire P. Abdominal Tuberculosis. J Assoc Physicians India. 2016;64(FEBRUARY):38-47.

4. Ladumor H, Al-Mohannadi S, Ameerudeen FS, Ladumor S, Fadl S. TB or not TB: A comprehensive review of imaging manifestations of abdominal tuberculosis and its mimics. Clin Imaging [Internet]. 2021;76(February):130-43. Available from: https://doi.org/10.1016/j.clinimag.2021.02.012

5. Abu-Zidan FM, Sheek-Hussein M. Diagnosis of abdominal tuberculosis: Lessons learned over 30 years: Pectoral assay. World J Emerg Surg. 2019;14(1):1-7.

6. Burrill J, Williams CJ, Bain G, Conder G, Hine AL, Misra RR. Tuberculosis: A radiologic review. Radiographics. 2007;27(5):1255-73.

7. Salooja BS, Mukhi S. Diagnostic value of computed tomography in the patients of abdominal tuberculosis. Int J Res Med Sci. 2016;5(1):206.

8. Kattan J, Haddad FG, Menassa-Moussa L, Kesrouani C, Daccache S, Haddad FG, et al. Peritoneal Tuberculosis: A Forsaken Yet Misleading Diagnosis. Case Rep Oncol Med. 2019;2019:1-4.

9. Chakaya J, Khan M, Ntoumi F, Aklillu E, Fatima R, Mwaba P, et al. Global Tuberculosis Report 2020 Reflections on the Global TB burden, treatment and prevention efforts. Int J Infect Dis. 2021;(xxxx):4-9.

10. Salvador F, Los-Arcos I, Sánchez-Montalvá A, Tórtola T, Curran A, Villar A, et al. Epidemiology and diagnosis of tuberculous lymphadenitis in a tuberculosis low-burden country. Med (United States). 2015;94(4):3-8.

11. Al-Zanbagi AB, Shariff MK. Gastrointestinal tuberculosis: A systematic review of epidemiology, presentation, diagnosis and treatment. Saudi J Gastroenterol. 2021;27(5):261-74.

12. Wang Z, Liu JC, Chen QL, Wu QH, Zhang Y. A LIP Abdominal Tuberculosis Classification System for Surgery. Indian J Surg. 2021;83(1):100-6.

13. Ha HK, Ko GY, Yu ES, Yoon KH, Hong WS, Kim HR, et al. Intestinal tuberculosis with abdominal complications: Radiologic and pathologic features. Abdom Imaging. 1999;24(1):32-8.

14. Bertlich M, Paparoupa M, Weckauf H, Wittig A, Linnemüller S, Schuppert F. The diagnostic challenge of abdominal tuberculosis in nonendemic countries: A case series from a tertiary hospital in Germany. Clin Case Reports. 2021;9(7):1-6.

15. Gupta P, Kumar S, Sharma V, Mandavdhare H, Dhaka N, Sinha SK, et al. Common and uncommon imaging features of abdominal tuberculosis. J Med Imaging Radiat Oncol. 2019;63(3):329-39.

16. Park JJ, Cheon JH. Atlas of Inflammatory Bowel Diseases. Atlas Inflamm Bowel Dis. 2015;71-85.

17. Lewis O, Tammana S, Sealy P. Peritoneal Tuberculosis: Looking beyond the Typical Pathology. Open J Intern Med. 2014;04(01):1-6. 